\title{
Identification of photoluminescence features of an epoxy resin based on components features and curing effects
}

\author{
O. Gallot-lavallée ${ }^{1 *}$, G. Teyssedre ${ }^{1}$, C. Laurent ${ }^{1}$ and S. Rowe ${ }^{2}$ \\ ${ }^{1}$ Université Paul Sabatier, Laboratoire de Génie Electrique de Toulouse, \\ 118, route de Narbonne, Toulouse, 31062, France \\ ${ }^{2}$ Schneider Electric, Direction des Recherches Matériaux, \\ Rue Henri Tarze, Grenoble, 38050, France \\ *E-mail : olg1@free.fr
}

Relevant section in Polymer:

PACS Numbers:

78.55.-m Photoluminescence, properties and materials

78.60.Kn Thermoluminescence

82.35.-x Polymers: properties; reactions; polymerization 


\begin{abstract}
Photoluminescence features in a commercial grade cured DGEBA-based epoxy resin have been investigated with as objective to determine which of the material compounds are responsible for cured resin emissions. The origin of the bands has been approached by considering photoluminescence of base resin and hardener taken separately, of their mixture, and in the course of curing. Most of the bands observed in the cured resin could be interpreted based on those found in the components taken separately. Fluorescence of the cured resin appears dominated by two broad bands that are characteristic of the hardener and exhibits a blue shift by up to $30 \mathrm{~nm}$ in the course of curing which could be used for cure monitoring purpose of the investigated system. Fluorescence of the base resin is clearly detected in the resin cured with tertiary amine as catalyzer. However, it is very weak if the catalyzer is not added. Two phosphorescence processes have been isolated in the cured resin, one of them being related to the base resin. Finally, a chemiluminescence spectrum has been recorded in the course of curing, which has been related to either curing-related reactions or to an oxidation process.
\end{abstract}

\title{
Keywords
}

Photoluminescence; Curing; Epoxy resin 


\section{Introduction}

Since their appearance, around 50 years ago, epoxy resins have been extensively used in electrical engineering industry [1]. Applications in this domain concern e.g. circuit-boards but also systems where good electrical insulation properties are combined to in-situ polymerization, such as solid insulation transformers, bus bar and insulation of electrical machines. Even though application of epoxy as electrical insulation has a long history, little is known about electrical ageing in this kind of material.

We have initiated a study of electrical ageing precursors in epoxies by means of space charge and conduction measurements, along with electroluminescence measurements $[2,3]$. Electroluminescence refers to light emission processes excited by interaction between electrical charges and the material. As usually when dealing with luminescence of polymeric materials, the interpretation of emission spectra in terms of molecular species is by no way a straightforward job. This is particularly true when considering epoxies since luminescence of the cured resin may arise from the complex and often only partially documented formulation of the main compounds that are hardener and base resin, as well as from molecular structures formed during the curing itself. It is therefore advisable to have as much information as possible on the photophysical behaviour of the resin for further understanding of its potential evolution under electric field.

The present paper aims at discussing the photoluminescence spectra of a cured epoxy resin based on the signatures found in the related compounds and on the evolution of the spectra during curing of the resin.

\section{Experimental Section}

\subsection{Materials}

The investigated resin corresponds to a commercial formulation [4] composed of a Diglycidyl ether of bisphenol A type epoxy resin, an anyhdride hardener based on methyl tetrahydrophtalic anhydride and a catalyst of the tertiary amine type. When mixing an equal weight of hardener and base resin and a small quantity of catalyser, an epoxy resin with a relatively low glass transition temperature $(\mathrm{Tg})$ of $65^{\circ} \mathrm{C}$ is obtained, due to the presence of a plasticizer in the hardener. 
The known chemical properties of the components are given in Table 1, where DGEBA means Diglycidyl ether of bisphenol A and MTHPA means Methyltetrahydrophtalic anhydride. These analyses were carried out essentially by gel permeation chromatography (GPC) and FTIR spectroscopy [5,6] The main components of the hardener are MTHPA at about 50\% [4] and a plasticizer, di-ester di-carboxylic acid at about 40\%. The remaining 10\% is not well documented and appears to contain some amine. As regards the base resin, it is essentially constituted by DGEBA-based groups. The catalyser is a tertiary amine. A typical reaction pathway (excluding etherification process) for curing of the epoxy resin is shown in Table 2. It consists in a three-step process with (1) initiation of the reaction through activation of the anhydride by the tertiary amine; (2) propagation by an esterification process between epoxy and carboxylate; (3) catalyser restitution.

The first kind of sample investigated was an epoxy resin cured in conditions similar to those used when moulding solid insulation transformers at industrial plant. The process consists in outgassing the components, hardener, and resin, taken separately, under primary vacuum, in order to remove water and air dissolved in it. These components plus catalyser are mixed and outgassed again and the mixture is cured in air for $16 \mathrm{~h}$ at $100^{\circ} \mathrm{C}$. Samples were cured in the form of disks having $500 \mu \mathrm{m}(+/-10 \mu \mathrm{m})$ thickness and $110 \mathrm{~mm}$ diameter. It will be referred to as ex-situ cured resin.

We also investigated base resin and hardener taken separately and after mixture (without catalyser), by photoluminescence (PL). As samples are in a viscous state, they were put in a crucible of $3 \mathrm{~mm}$ thickness and $55 \mathrm{~mm}$ diameter. Finally, PL was measured during thermostimulation, i.e. curing of the mixture, in air. The material obtained after $18 \mathrm{~h}$ of curing at $100^{\circ} \mathrm{C}$ will be referred to as in-situ cured resin.

\subsection{Characterisation}

The home-made chamber and its main components we used for PL measurements are depicted in Figure 1. A cooled Photo Multiplier (PM) is used to integrate the number of detectable photons with a variable time resolution, i.e. dwelling time (10 $\mu$ s up to some minutes). In the present experiments it was used for the purpose of phosphorescence lifetime measurement. Emission spectra are acquired by means of a cooled Charged Coupled Device (CCD) camera coupled to a grating monochromator, with a detection wavelength range of $190 \mathrm{~nm}$ to $820 \mathrm{~nm}$ and a resolution of $3 \mathrm{~nm}$. The excitation setup is built on a $150 \mathrm{~W}$ Xenon 
lamp coupled to a monochromator giving a range of excitation wavelength of $200 \mathrm{~nm}$ to $800 \mathrm{~nm}$. Except otherwise stated, intensities are provided in counts number, for an integration time of $5 \mathrm{~s}$ with the CCD camera.

The holder for solid samples and crucibles is thermally controlled $\left(-130^{\circ} \mathrm{C}\right.$ to $\left.100^{\circ} \mathrm{C}\right)$ by a feed back from a Pt100 temperature sensor installed in the holder. In these conditions, reasonable temperature control and homogeneity within the sample is obtained, even so there may be some temperature gradient in the system. PL measurements at room temperature provide information on fluorescence features of the material. Due to quenching by molecular oxygen, phosphorescence is usually not observed at room temperature. Therefore, experiments were realized at low temperature $\left(-130^{\circ} \mathrm{C}\right)$ for revealing phosphorescence processes. For these low temperature measurements, it is necessary to have a dry atmosphere. Helium was used at atmospheric pressure, providing also a good thermalization of the samples. The chamber was evacuated for $30 \mathrm{~min}$ under secondary vacuum $\left(10^{-6} \mathrm{mb}\right)$ prior to helium introduction. For all other experiments, the atmosphere was air. Indeed, changing the atmosphere requires previous evacuation of the chamber and this can not be achieved on liquids due to a high risk of pollution of the chamber. For this reason, we did not perform phosphorescence measurements on liquid samples.

PL emission spectra were obtained in 10nm intervals of the excitation wavelength, starting at typically $220 \mathrm{~nm}$ and ending in the range 300-400nm, depending on the response of the material. No correction for instrument response was applied to the spectra. While the response of the detection devices (PM and CCD) is relatively flat in the range 300-800nm, that of the Xenon lamp is not at all (excitation intensity increases by about 2 decades in the range 220$300 \mathrm{~nm}$ ). Hence, significant instrument response is introduced in the results, especially as regards excitation spectra. Spectra are therefore analyzed in relative terms only.

\section{Photoluminescence of cured resin and compounds}

\subsection{Photoluminescence of compounds}

Figure 2 shows the emission spectrum obtained on the base resin along with excitation spectra monitored at characteristic emission wavelengths. A main emission band is observed with maximum at $317 \mathrm{~nm}$, whatever the excitation wavelength. The corresponding excitation peak is found at about 290nm. This chromophore is defined as BR(290-317) (excitation at 290nm, emission at $317 \mathrm{~nm}$; BR stands for base resin). A second emission band is found at $383 \mathrm{~nm}$. 
The excitation spectra for the two bands are not parallel, indicating that the two emissions arise from different species. It is difficult, in case of the $383 \mathrm{~nm}$ band, to decide about the position of the excitation maximum. Hence we define this second band as BR(x-383).

Photoluminescence results for the hardener are shown in Figure 3, where the most representative features are produced. Two distinct emission processes are identified in the excitation and emission spectra. These are referred to as $\mathrm{H}(345-445)$ and $\mathrm{H}(396-487)$. A shoulder is clearly detected at $290 \mathrm{~nm}$ in the excitation spectra of the hardener. With this excitation wavelength, the emission is the superposition of the $445 \mathrm{~nm}$ broad band and some lower wavelength emissions.

\subsection{Photoluminescence of cured epoxy}

Figure 4 shows room temperature PL emission and excitation spectra of the ex-situ cured resin. Three emission bands are found at 312,431 and $460 \mathrm{~nm}$. The respective excitation bands are found at 287, 360 and $396 \mathrm{~nm}$. These processes are referred to as $\operatorname{ECR}(287-312)$, ECR(360-431) and ECR(396-460). ECR stands for ex-situ cured resin.

These emissions can readily be compared to those found in the compounds. Hence, we may infer that the component BR(290-317) found in the base resin is responsible for the lower wavelength contribution to PL of the ex-situ cured resin ECR (287-312), whereas the other two would be associated to the hardener though consistency in position of the bands is more evident for the longer wavelength one H(396-487) than for the other one H(345-445). Difference in the state of the sample (solid vs. liquid) along with possible curing effect could be responsible for the shift of the bands. This is why we consider in section 4 how PL evolves in the course of curing.

Low temperature measurements under continuous excitation (Figure 5) essentially reveal the same excitation spectra as those obtained at room temperature, along with broadly the same emission bands. The only difference is a growing up of the emission above c.a. $450 \mathrm{~nm}$ and an excitation shoulder appearing near $300 \mathrm{~nm}$. To resolve the underlying processes, we have acquired several emission spectra synchronized with excitation switch off using a mechanical shutter. In that way only long-lived emissions, i.e. phosphorescence processes, are detected. Figure 6 shows emission spectra so obtained. By using different excitation wavelengths, two phosphorescence processes are revealed. The best resolved band corresponds to an excitation of $300 \mathrm{~nm}$ and is found at $465 \mathrm{~nm}$. It is referred to as ECRp (300- 
$465)$ in the following. The second band, termed ECRp (340-495), is observed when exciting at $340 \mathrm{~nm}$, the emission maximum being at $495 \mathrm{~nm}$. The new excitation shoulder appearing in Fig. 5 when monitoring at either 427 or $463 \mathrm{~nm}$ is most probably related to the phosphorescence contribution ECRp (300-465) to the continuous wave emission.

Phosphorescence contributions produce an apparent broadening of the emission in respect to the room temperature data of Fig.4. In case of excitation at $290 \mathrm{~nm}$, the long-wavelength emission peak appears dominated by the phosphorescence at $463 \mathrm{~nm}$. Comparing emission spectra at $\lambda_{\text {exc }}=360 \mathrm{~nm}$ of Fig. 4 and 5, a slight broadening of the long-wavelength side of the emission spectrum is noticeable, which can be explained by the contribution of ECRp(340495).

As none of the main emission bands found under continuous excitation at room temperature appear in decay spectra, we can conclude on the fluorescence nature of ECR(287312), ECR(360-431) and ECR(396-460). Indeed, excitation at 340nm, i.e. in conditions where the bands $\operatorname{ECR}(360-431)$ and $\operatorname{ECR}(396-460)$ can normally be seen under continuous excitation lets appear only an emission at nearly 500nm. Overall, these low temperature measurements allow us to conclude on the fluorescence nature of emission processes found at room temperature and the phosphorescence nature of $\operatorname{ECRp}(300-465)$ and $\operatorname{ECRp}(340-495)$.

Another way of revealing phosphorescence processes consists in acquiring delayed emission with the photomultiplier, at either excitation switch-on or switch-off. In the hypothesis of a delayed emission, from a single specie, with apparent lifetime $\tau$, light emission intensity at excitation switch-on and switch-off should obey [7]:

$$
I_{o n}=I_{o}+I_{d}\left[1-e^{-t / \tau}\right] \quad \text { and } \quad I_{o f f}=I_{d} \cdot e^{-t / \tau}
$$

where $I o$ and $I d$ are intensities for instantaneous (e.g. fluorescence) and delayed processes, respectively. We recorded the phosphorescence build-up and decay while exciting at $260 \mathrm{~nm}$, i.e. in conditions where the ratio of phosphorescence-to-fluorescence was the highest. Fitting to Equation 1 leads to an apparent lifetime of triplet state $\tau \approx 0.9 \mathrm{~s}$. A trace of this recording is shown in Figure 7.

\subsection{Discussion}

The chromophore ECR(287-312) has been tentatively attributed to the base resin, which is constituted mainly by DGEBA. Xu et al. [8] have investigated the photoluminescence of a 
BisPhenol A DiCYanate Ester (BPADCY) resin, and have considered the effect of addition of BisPhenol A to the resin. They reported luminescence of the latter at $(\lambda \mathrm{exc}=265 \mathrm{~nm}$ $\lambda$ emi $=305 \mathrm{~nm}$ ). According to Bridges [9], the fluorescence features of biphenyl are: $(\lambda$ exc $=246$ to $260 \mathrm{~nm}-\lambda$ emi $=318 \mathrm{~nm})$ and those of phenol are: $(\lambda$ exc $=272 \mathrm{~nm}$ $\lambda$ emi. $=320 \mathrm{~nm}$ ). These results confirm the hypothesis on the origin of the fluorescence band of the cured resin, $\operatorname{ECR}(287-312)$, as related to the base resin, and show that bisphenol is likely the emitting group. In addition, Griseri [10] has investigated the photoluminescence properties of a DGEBA-based resin whose spectral signatures are close to those presented therein. Indeed, fluorescence bands are reported at $(\lambda$ exc $=305 \mathrm{~nm}-\lambda$ emi $=325 \mathrm{~nm}) ;(318-375 \mathrm{~nm})$ and (353-400nm). Again, the features of the main band in their spectrum (the lower wavelength one) appear consistent with those of $\operatorname{ECR}(287-312)$. The other emissions found by Griseri \& al. have been attributed to an amine. These emissions might be related to $\mathrm{BR}(\mathrm{x}-383)$ found in the actual work, which would be consistent with the presence of an amine in our base resin.

Concerning fluorescence emissions found in the cured resin and attributed to the hardener, it is difficult to infer their molecular origin, given the lack of reference photoluminescence data on hardener's components.

As regards phosphorescence results, Griseri et al. [11] reported a structured emission in a cured DGEBA-based epoxy resin. At least two phosphorescence processes were identified, one with emission maximum at about $450 \mathrm{~nm}$, and the other with maxima at 510 and $550 \mathrm{~nm}$, with excitation respectively at about 350 and 400nm. Also, the phosphorescence properties of an epoxy resin film was examined and compared with the corresponding properties of the uncured resin in solution by Allen et al. [12]. At relatively low concentration of base resin (e.g. $5 \%$ ), the monomeric emission at $405 \mathrm{~nm}$ of Bisphenol $\mathrm{A}$ is replaced by a longer wavelength emission at $460 \mathrm{~nm}$, together with a corresponding shift in the excitation spectrum, from 275 to $350 \mathrm{~nm}$. The polymer phosphorescence has been associated with the presence of an associated ground-state aggregate and triplet excimer [12]. The important point here is that the phosphorescence emission at $465 \mathrm{~nm}$ reported in Figure 6 for the cured resin is most probably characteristic of the base resin. As regards the other band found in decay spectra of Figure 6 (495nm when exciting at $340 \mathrm{~nm})$, no direct comparison can be made with results of Griseri. It is worth stressing that their hardener provided different signature from those reported therein, and thus it is not unexpected that different luminescence properties be obtained for the respective cured resins. 
To conclude, most of the emission bands found in the cured resin could be explained based on the emission features of the main components that are base resin and hardener. We have given evidence of the fluorescence nature of $\operatorname{ECR}(287-312)$, due to bisphenol from base resin, phosphorescence nature of ECRp (300-465) related to the base resin and finally fluorescence of the hardener, which provides ECR(360-431) and ECR(396-460) features. Except, perhaps, for the last phosphorescent band ECRp (340-495) which could not be attributed to a specific compound due to a lack of reference data, we have no evidence of new luminescent species formed by curing.

\section{Photoluminescence during curing}

\subsection{Results}

We mixed in air the same proportion of hardener and base resin, without adding catalyser. Normalized PL spectra obtained during heating of the mixture are reported in Figure 8 whereas Figure 9 shows the time temperature cycle and the quantitative evolution of the emitted signal.

Spectra obtained at room temperature just after mixing (Figure 8.a) show maxima at 317 , 458 and 490nm when exciting at 290,360 and 400nm, respectively. As the mixture has a priori not reacted, one would expect to find the signatures of the compounds that are at $317 \mathrm{~nm}$ for the base resin and at 445 and $487 \mathrm{~nm}$ for the hardener. Clearly a red-shift of the emission formally at $445 \mathrm{~nm}$ is observed here, i.e. in the opposite way as when considering the cured resin (blue shift to $431 \mathrm{~nm}$ ). It is therefore wise to follow how this band evolves during curing.

In the course of heating to $100^{\circ} \mathrm{C}$ (Figure $8 \mathrm{~b}-\mathrm{d}$ ), the position of the bands did not evolve much. However, evolutions were observed in quantitative terms. The intensity of all the bands decreased, the relative decrease being the largest for the $317 \mathrm{~nm}$ band and the smallest for the 490nm one (cf. Figure 9). This decrease in emission intensity may correspond to a change in the photophysical properties of the mixture, i.e. emission yield can be temperature dependent. However, the change in intensity is relatively strong, especially for the band at $317 \mathrm{~nm}$. It is therefore thought that ongoing reactions produce such changes. This is confirmed by considering the results obtained at $100^{\circ} \mathrm{C}$ at $5800 \mathrm{~s}$. The band at $317 \mathrm{~nm}$ has further decreased, by over $60 \%$, in respect to the value at 940 s. Curing therefore has as major effect to make decrease the fluorescence intensity related to the base resin. Considering features related to 
the hardener, the band at $460 \mathrm{~nm}$ does not change in position whereas that at $490 \mathrm{~nm}$ is blue shifted to $480 \mathrm{~nm}$ at time $5800 \mathrm{~s}$.

Another noticeable features in all the spectra presented in Figure 8 is a shoulder at 540$545 \mathrm{~nm}$ that was observed neither in the spectra of the cured resin nor in those of the components taken separately. This peak is detected just after mixture of base resin and hardener, grows with temperature and time and apparently decreases at long time at $100^{\circ} \mathrm{C}$.

For the test realized previously, the sample was kept at constant temperature $\left(100^{\circ} \mathrm{C}\right)$ for 5 hours. After the experiment, the resin was in a rubber-like state, i.e. it is clear that curing was not completed. Therefore, we repeated curing in the same conditions on another mixture, waiting for $18 \mathrm{~h}$ at $100^{\circ} \mathrm{C}$. PL spectra obtained after cooling to $40^{\circ} \mathrm{C}$ are plotted in Figure 10, and a corresponding data point has been added in Figure 9. The band at $317 \mathrm{~nm}$ has still undergone a decrease, relatively to the other bands, if compared to results shown in Figure 8.e. The decrease of absolute intensity of this band is evident in Fig.9. Other trends are observed, that are a blue shift of the two bands associated to the hardener, and the disappearance of the shoulder at $545 \mathrm{~nm}$. The peak wavelengths of the bands related to the hardener are still half-way if compared to their position in the cured resin of Figure $4 \mathrm{a}$.

\subsection{Discussion}

PL data obtained in the course of curing allow us to build a more complete frame of the PL features of cured resin. One of the fluorescence bands associated to the hardener $\mathrm{H}(345-445)$ exhibits an important red-shift (by $15 \mathrm{~nm}$ ) just after mixture at $20^{\circ} \mathrm{C}$ with the base resin. This effect can not be explained by a reaction process, since qualitatively, no more changes are observed for this band when heating up to $100^{\circ} \mathrm{C}$. It is likely related to some solvent effect on the species at the origin of this process. Curing has as effect to shift the peak wavelength from $460 \mathrm{~nm}$ after mixture, to $450 \mathrm{~nm}$ after $18 \mathrm{~h}$ of curing at $100^{\circ} \mathrm{C}$ without catalyser and to $431 \mathrm{~nm}$ when curing with catalyser. The band H(396-487), also related to the hardener, does not appear sensible to mixture with the base resin. It is blue-shifted from $490 \mathrm{~nm}$ to $480 \mathrm{~nm}$ after $1 \mathrm{~h} 30$ at $100^{\circ} \mathrm{C}$ and to $474 \mathrm{~nm}$ after $18 \mathrm{~h}$ at $100^{\circ} \mathrm{C}$, both conditions being without catalyser, and to $460 \mathrm{~nm}$ when curing in presence of catalyser. In quantitative terms, it appears that the two hardener-related fluorescence signatures decrease in the course of heating of the mixture. However, this feature can be partly due to temperature effect on the fluorescence response, and not enough data are available as a function of time in isotherm conditions for allowing 
reliable conclusions to be drawn. Curing therefore has as principal consequence to blue-shift hardener-related emissions. This shift is less important when considering the resin cured insitu as compared to ex-situ curing, for comparable curing time. This is not unexpected since the latter has been cured in presence of the catalyser, so that the curing kinetics is normally faster. Hence, the resin cured in-situ would be only partially cured.

As regards results for the fluorescence band associated to the base resin BR(290-317), the trend is relatively clear considering in-situ curing data. A marked decrease of the intensity is observed in the course of heating, and the emission becomes very small in comparison to hardener signature after $18 \mathrm{~h}$ at $100^{\circ} \mathrm{C}$. Curing effects would therefore consist in a decrease of the intensity of this emission, along with a small red-shift. This is however not confirmed when considering fluorescence of the ex-situ cured resin (Fig.4). In that case, the emission is found at $312 \mathrm{~nm}$ (blue shifted in respect to base resin or mixture features) and the intensity is strong relatively to hardener's signature (cf. spectra for excitation at 290nm). Differences in curing conditions between the two sets of data concern the presence of catalyser on the one hand, giving possibly a different network structure, and the fact that in-situ curing was made without previous outgassing of the components on the other hand, this latter feature rendering possible that some oxidation processes be at play. Concerning the first point, it was reported than polymerization of anhydride-epoxies systems without catalyzer involves an etherification reaction, giving rise to homopolymerisation of the resin, which competes with the esterification process $[13,14]$. Whereas the introduction of amine catalyzer gives rise exclusively to esterification, it was observed that etherification occurs for the investigated system without catalyzer [5]. Curing without catalyzer therefore leads to longer linear chain segments.

Several methods for in situ monitoring cure reactions in DGEBA-based epoxies have been proposed, using either intrinsic fluorescence or fluorescence from reactive labels as extrinsic fluorophors $[15,16]$. From the above results, monitoring of curing could be achieved by means of either monitoring of the position of the hardener-related fluorescence bands (qualitative analysis which would not require calibration for quantity analyzed), or from monitoring of the fluorescence at $317 \mathrm{~nm}$, but in this case changes in the response due to change in the curing conditions (catalyser and/or atmosphere) must be analyzed in detail.

The last feature we would like to stress upon is the appearance of a shoulder on the long wavelength side of PL spectra obtained during curing. The band is detected just after mixture of hardener and base resin; it is maximum when the temperature reaches $100^{\circ} \mathrm{C}$ and seems 
decreasing after $1 \mathrm{~h} 30$ at $100^{\circ} \mathrm{C}$. It is no longer observed in either in-situ cured resin (Figure 10) or in ex-situ cured epoxy (Figure 4). These experiments have been complemented by acquisition of the spectrum of spontaneous luminescence during curing (no UV excitation). Figure 11 shows the spectrum obtained by integrating light for 5 min with the CCD camera once temperature was stabilized to $100^{\circ} \mathrm{C}$. The emission maximum is found at about $560 \mathrm{~nm}$, i.e. not so far from the shoulder at $545 \mathrm{~nm}$ in PL spectra. Note that in Figure 8, the wavelength of the shoulder is underestimated since it is superimposed to a broad band peaking at $490 \mathrm{~nm}$. The first interpretation would be that base resin and hardener combination produces intermediate chemicals during reaction, and these chemicals would exist only during the reaction phase. They could be detected by photoluminescence (being excited through either direct excitation or energy transfer from other groups), or by spontaneous luminescence since reactions often provide intermediate (or final) products in the excited state, their relaxation giving rise to light emission. However, a cured resin previously stored in ambient conditions may also give rise to luminescence during thermostimulation $[3,17]$. We have shown that the spectrum of this light is the same as that obtained during curing [18]. This phenomenon is dependent on the nature of the atmosphere (nitrogen or air, humidity or not...) and on previous storing conditions. It is therefore not clear whether it corresponds to curing / post curing or to an oxidation effect $[19,20]$. We address more extensively this point in a forthcoming paper [18].

\section{Conclusion}

The emission bands found in a commercial-grade cured epoxy resin could be explained based on the emission features of the DGEBA-based resin and hardener. The base resin exhibits a fluorescence process at $\lambda_{\mathrm{exc}}=287 \mathrm{~nm} / \lambda_{\mathrm{exc}}=312 \mathrm{~nm}$, attributed to the bisphenol. This emission appears to be decreasing in the course of curing, except for the case of the resin cured in presence of an amine-based catalyser in which case the emission is still relatively strong. The hardener, constituted mainly by MTHPA, appears responsible for the main

fluorescence bands of the cured resin: $\lambda_{\mathrm{exc}}=360 \mathrm{~nm} / \lambda_{\mathrm{exc}}=431 \mathrm{~nm}$ and $\lambda_{\text {exc }}=396 \mathrm{~nm} / \lambda_{\text {exc }}=460 \mathrm{~nm}$. A red-shift of these emissions was observed when the hardener was mixed to the base resin, presumably due to a solvatochromic effect. In the course of curing, these emissions shifted to the blue. Among parameters considered here, i.e. emission intensities and position of the various bands, the variation of the maximum wavelength of the hardener fluorescence emissions appears as the most suited for monitoring curing of the 
investigated system. Spontaneous luminescence was detected in the course of curing and the corresponding spectrum has been recorded. At the present stage it is not clear whether the chemiluminescence being measured results from curing-related reactions or if it corresponds to an oxidation process.

\section{Acknowledgments}

Thanks are due to François Trichon, Stéphane Robiani, Jacques Gatellet and Valérie Ollier-Durault who kindly provided information on the investigated system. 


\section{References and notes}

[1] Dakin TW. IEEE Trans. Diel. \& Elec. Insul. 1974; 9:121-128.

[2] Gallot-Lavallee O, Teyssedre G, Laurent C, Rowe S. Annual Report Conf. on Electrical Insulation and Dielectric Phenomena, Albuquerque, NM, USA, 2003, pp.249-252.

[3] Gallot-Lavallee O, Teyssedre G, Laurent C, Rowe S. Proc. International Conference on Solid Dielectrics, Toulouse, France, July 2004. pp. 703-706.

[4] Commercial products are referenced as HY227 for the hardener, CY225 for the base resin resin and DY062 by Ciba Geigy (now Huntsman)

[5] Ollier-Dureault V. Vieillissement de surfaces d'isolateurs en résine époxy-anhydride: rôles respectifs des UV et du champ électrique. Thesis, Grenoble (Fr): Université Joseph Fourier, 1995.

[6] Ollier-Durault V, Gosse B. J. Appl. Polymer Sci. 1998; 70:1221-1237.

[7] Teyssedre G, Menegotto J, Laurent C. Polymer 2001; 42:8207-8216.

[8] Xu YE, Sung CSP. Macromolecules 2002; 35:9044-9048.

[9] Bowen EJ. Luminescence in Chemistry, London: van Nostrand Company ltd, 1968.

[10] Griseri V. The effects of high electric fields on an epoxy resin. Ph D Thesis, Leicester: University of Leicester (UK), 2000.

[11] Griseri V, Dissado LA, Fothergill JC, Laurent C, Teyssedre G. J. Phys. D: Appl. Phys. 2001; 34:2534-2540.

[12] Allen NS, Binkley JP, Parsons BJ, Phillips GO, Tennant NH. Polymer Photochemistry $1982 ; 2: 389-393$.

[13] Fischer RF. J. Polym. Sci. 1960; 44:155-172.

[14] Stevens GC. J. Appl. Polymer Sci. 1981; 26:4258-4278.

[15] Mikes F, Gonzales-Benito F, Serrano B, Bravo J, Baselga J. Polymer 2002; 43:43314339 .

[16] Sung CSP, Sung NH. Materials Sci. Engineering 1993; A162:241-247.

[17] Suzuki T, Sunose T, Amasaki I, Ozawa T. Polymer Degradation and Stability 2002; 77:87-91.

[18] Gallot-Lavallee O, Teyssedre G, Laurent C, Rowe S. J. Appl. Polymer Science, under submission.

[19] Lee H, Neville K. Handbook of epoxy resins, New York: McGraw-Hill, 1967.

[20] Le Huy HM, Bellenger V, Paris M, Verdu J. Polymer Degradation and Stability 1992; $35: 77-86$. 


\section{Figure captions}

Figure 1: Schematic of the photoluminescence experimental set-up.

Figure 2: Room temperature photoluminescence spectra of the base resin. (a): Emission spectrum; (b): Excitation spectra

Figure 3: Room temperature photoluminescence spectra of the hardener. (a): Emission spectra; (b): Excitation spectra

Figure 4: Room temperature photoluminescence spectra of the ex-situ cured resin (a): Emission spectra; (b): Excitation spectra.

Figure 5: Low temperature photoluminescence $\left(-130^{\circ} \mathrm{C}\right)$ spectra of the ex-situ cured resin (a): Emission spectra; (b): Excitation spectra.

Figure 6: Low temperature phosphorescence spectra of ex-situ cured resin.

Figure 7: Luminescence transients on ex-situ cured resin after excitation at 260nm and after switching off the excitation $\left(\mathrm{T}=-130^{\circ} \mathrm{C}\right)$. The photomultiplier dwelling time was $100 \mathrm{~ms}$. Fitting parameters to Eq. 1 are given in inset.

Figure 8: Normalized PL spectra recorded during in-situ curing

Figure 9: Emission intensities monitored at 317, 460 and 490nm for excitation respectively at 290, 360 and 400nm and corresponding temperature cycle.

Figure 10: Normalized emission spectra of the resin cured in-situ for $18 \mathrm{~h}$ at $100^{\circ} \mathrm{C}$.

Figure 11: Spontaneous emission spectrum of the resin obtained during in-situ curing at $100^{\circ} \mathrm{C}$. The spectrum has been integrated over $5 \mathrm{~min}$ starting at the time the temperature set point was reached. The solid line is a smoothing of data. 


\section{Table}

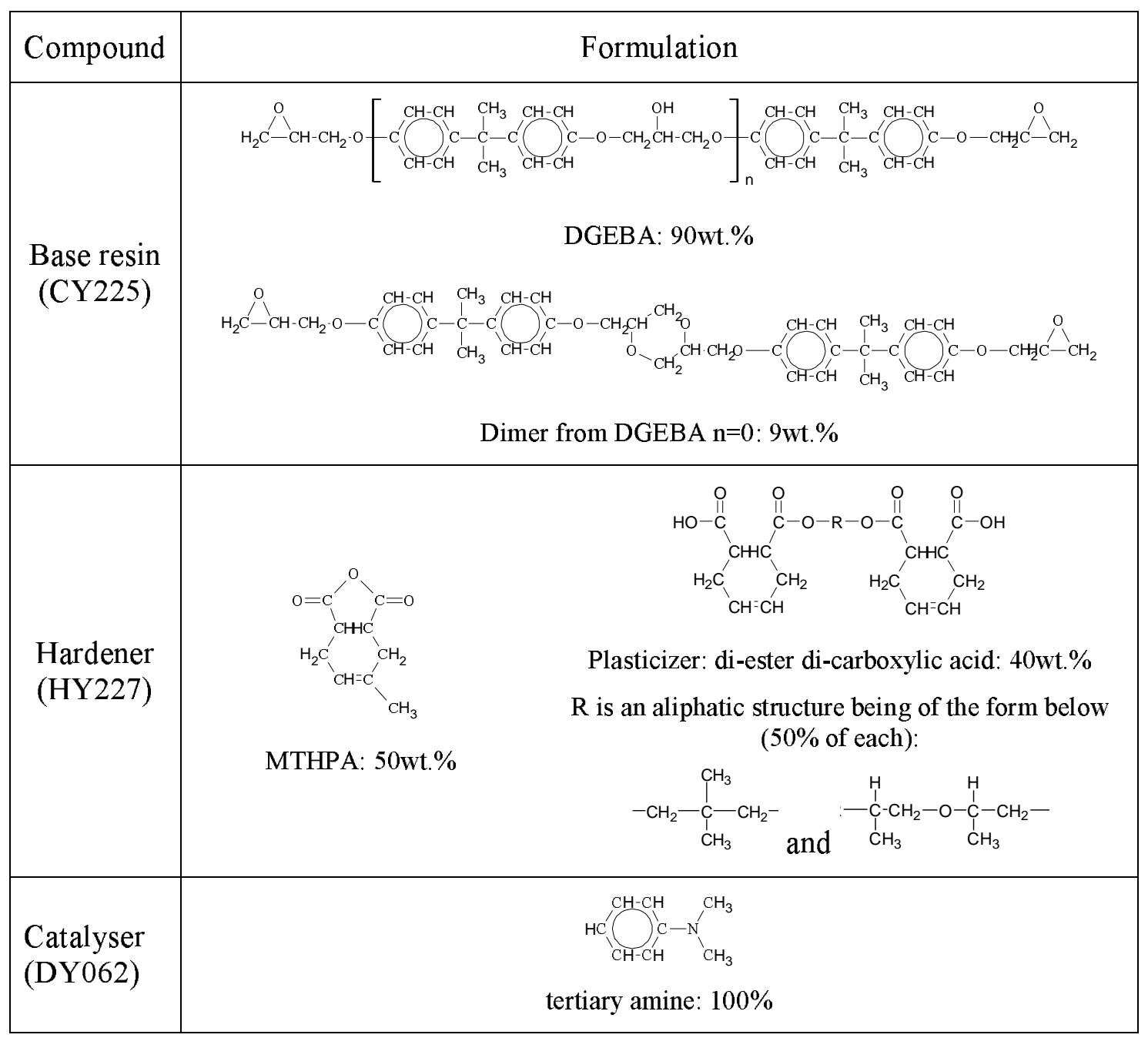

Table 1: Known chemical formulation of components of the resin. $10 \%$ of the hardener content is unknown $[5,6]$. 


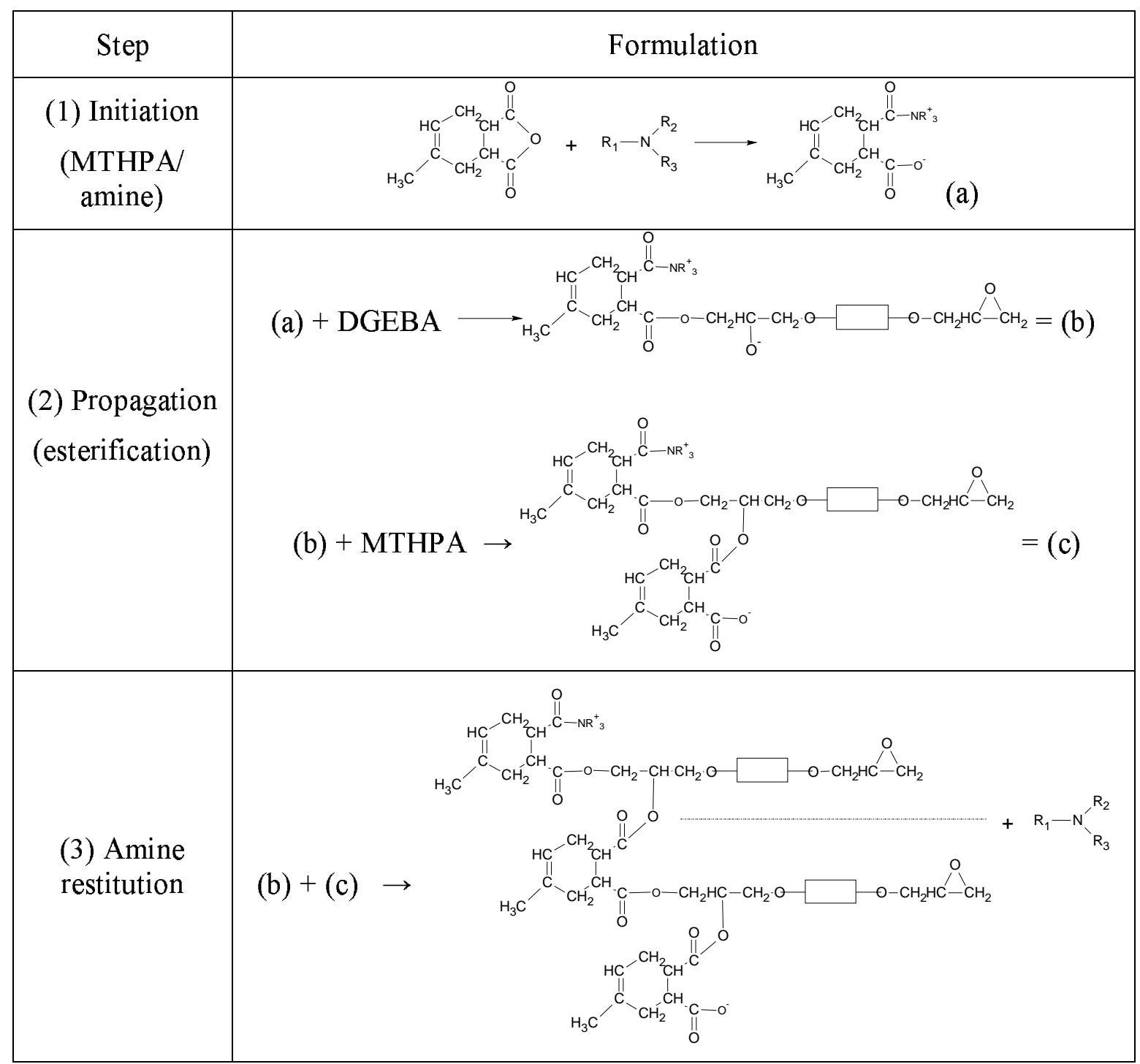

Table 2: Ideal reaction pathway for epoxy polymerization. 


\section{Figures}

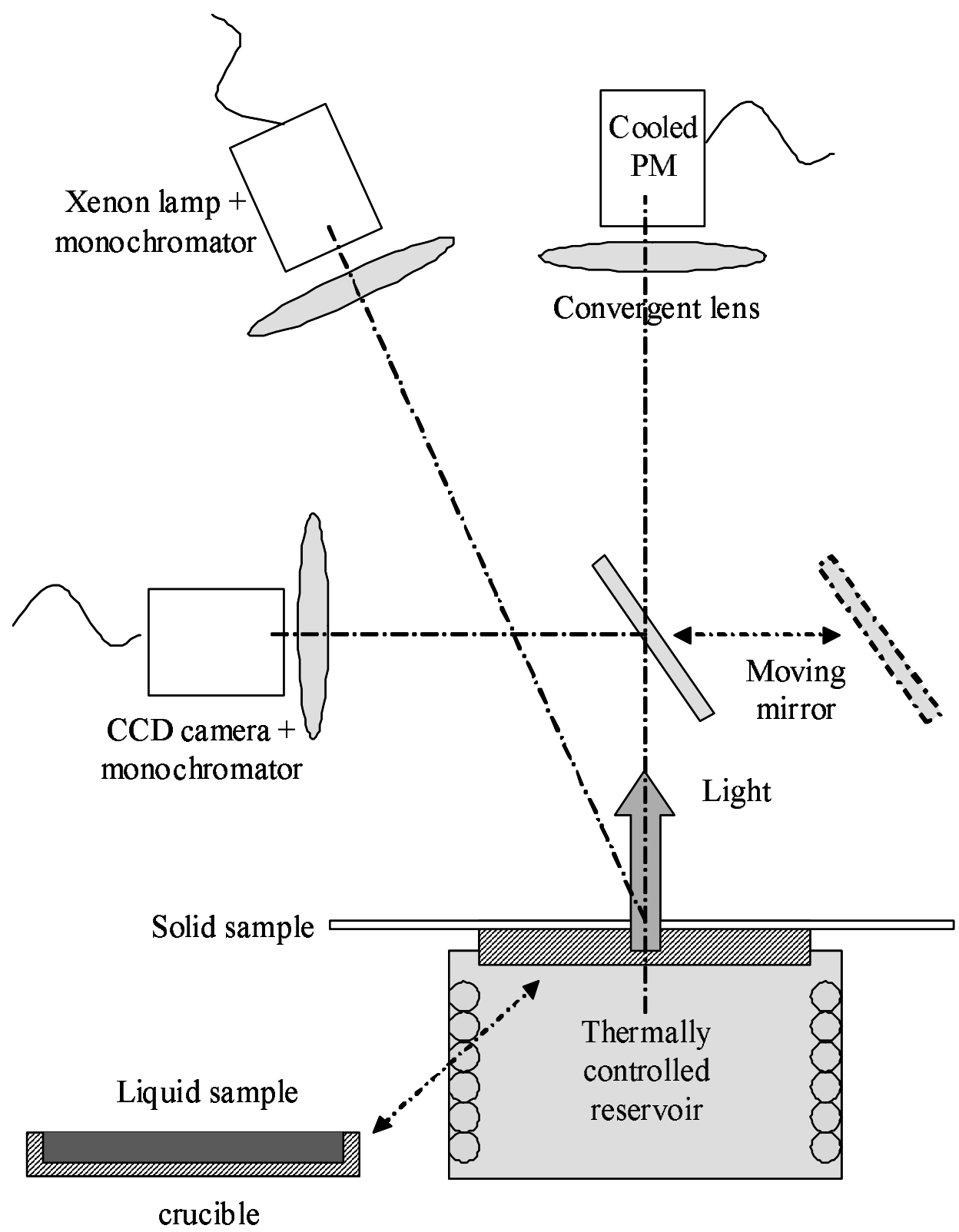

Figure 1: Schematic of the photoluminescence experimental set-up. 

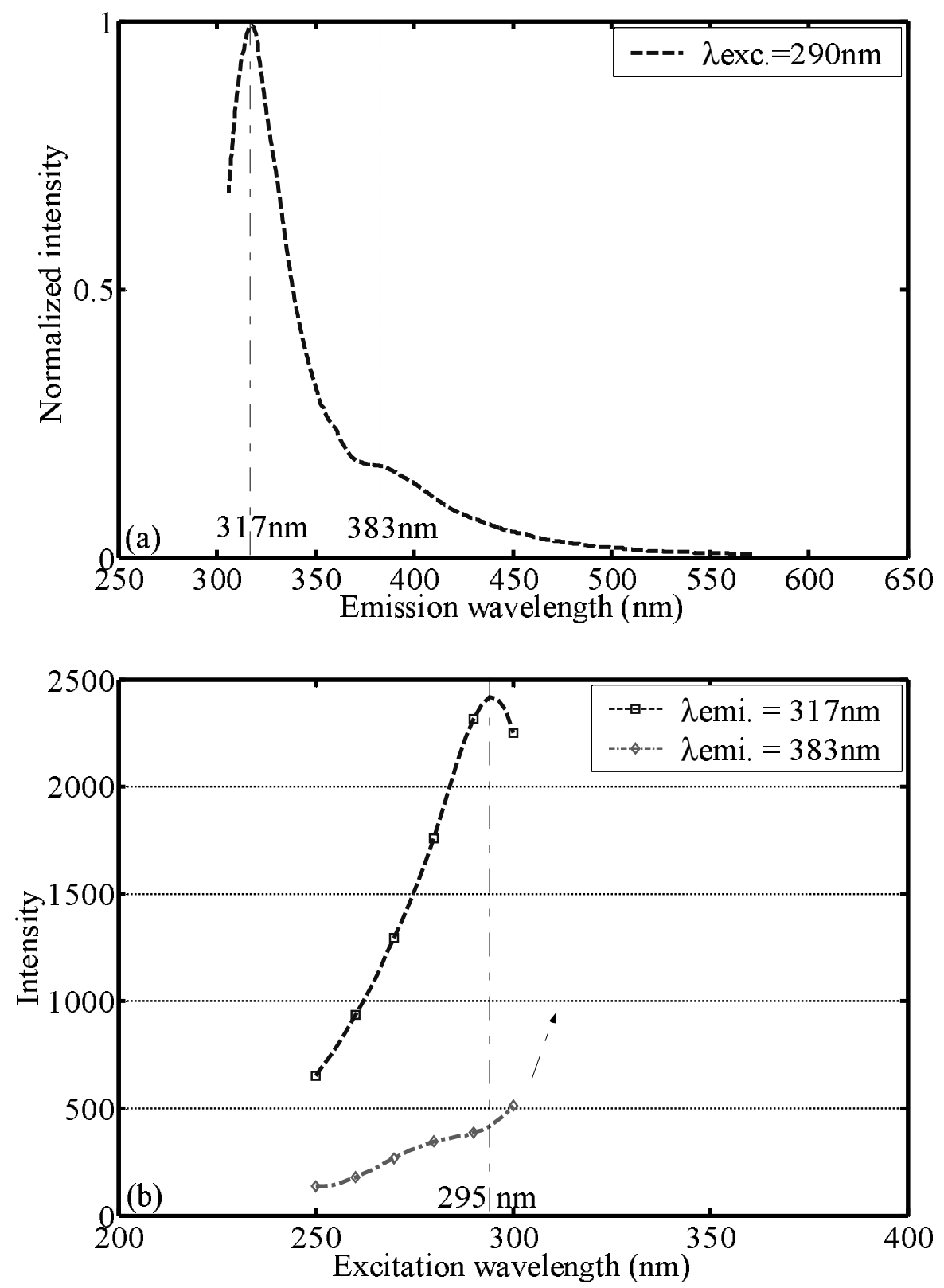

Figure 2: Room temperature photoluminescence spectra of the base resin. (a): Emission spectrum; (b): Excitation spectra 

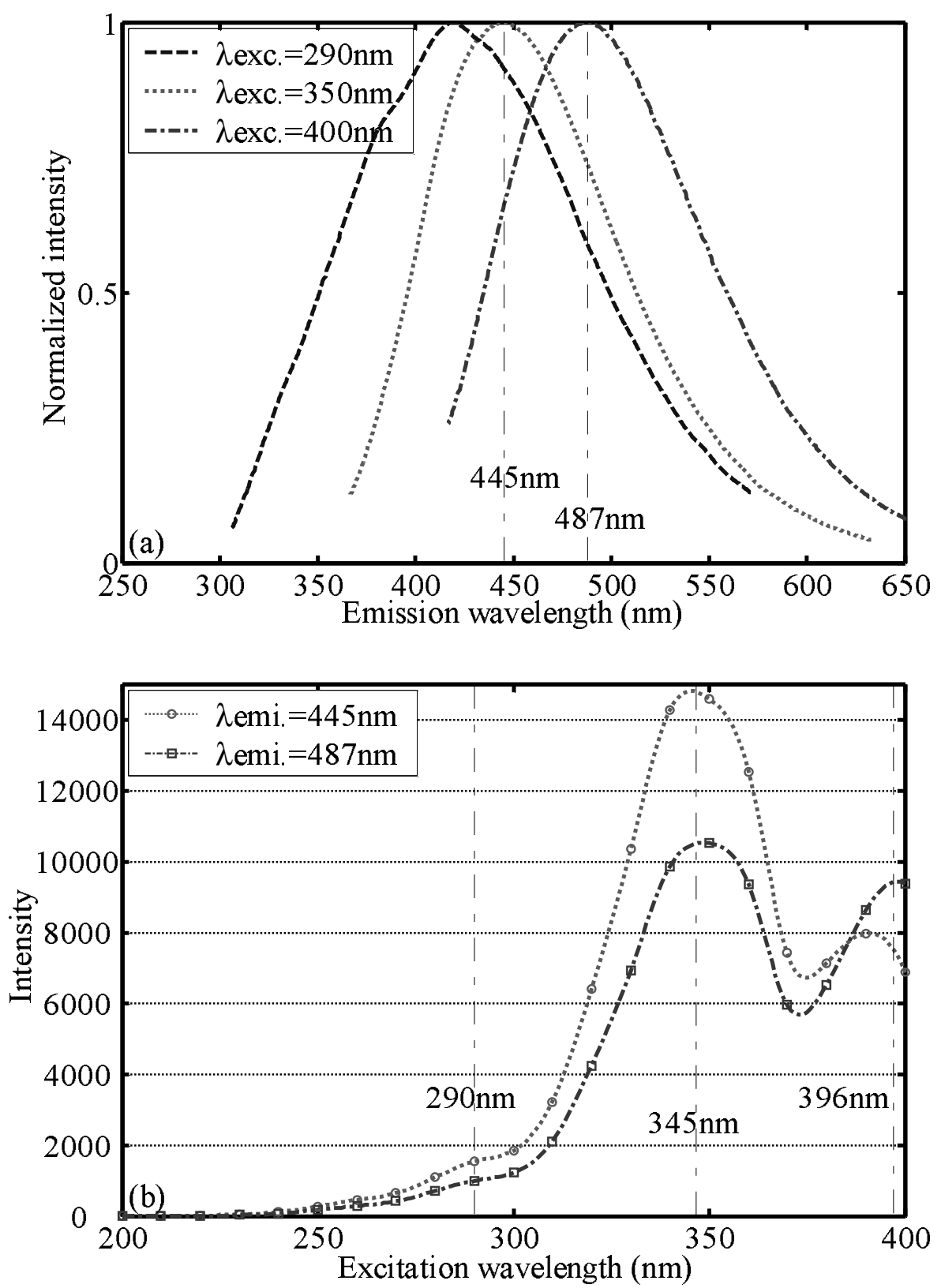

Figure 3: Room temperature photoluminescence spectra of the hardener. (a): Emission spectra; (b): Excitation spectra 

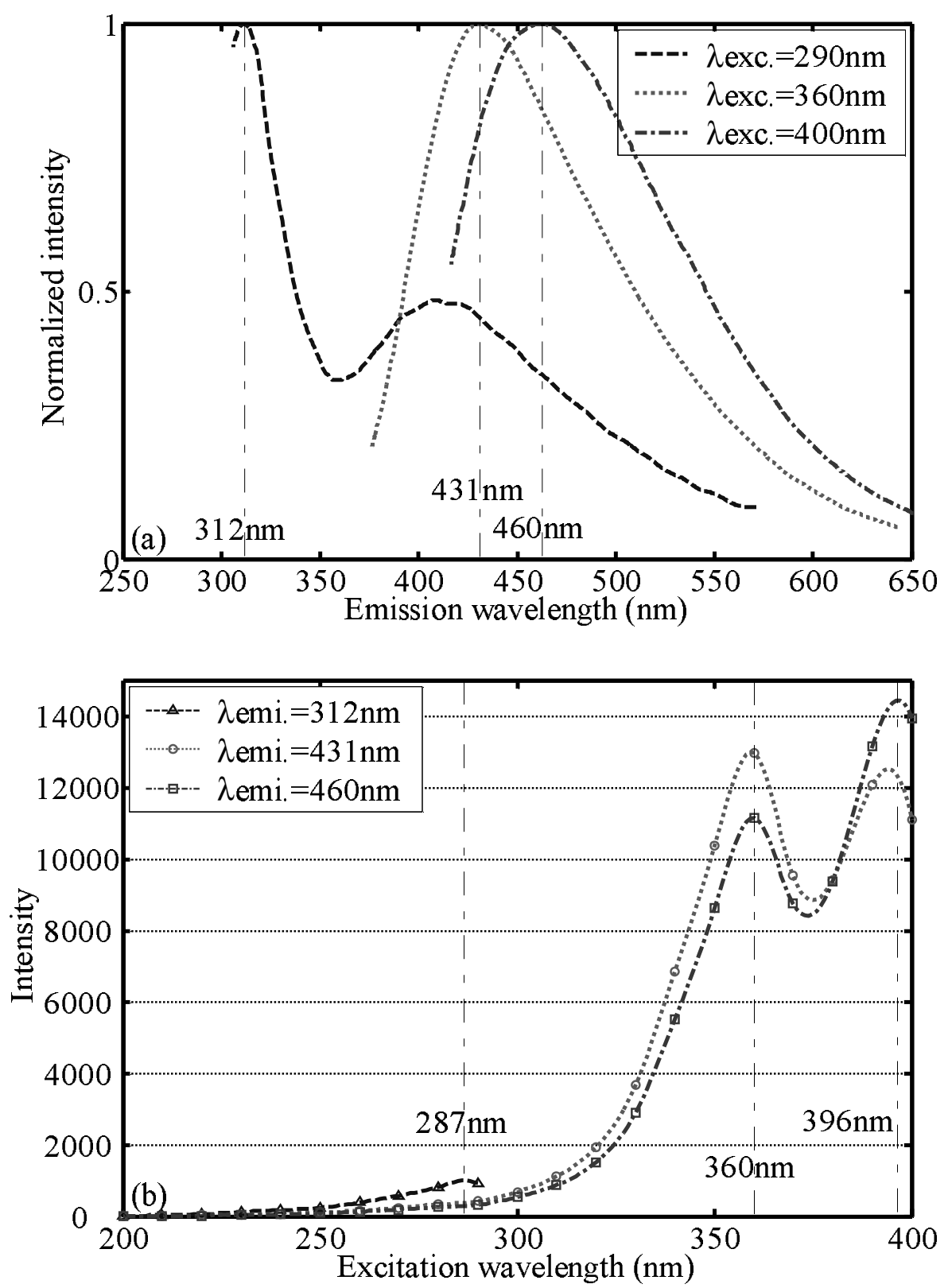

Figure 4: Room temperature photoluminescence spectra of the ex-situ cured resin (a): Emission spectra; (b): Excitation spectra. 

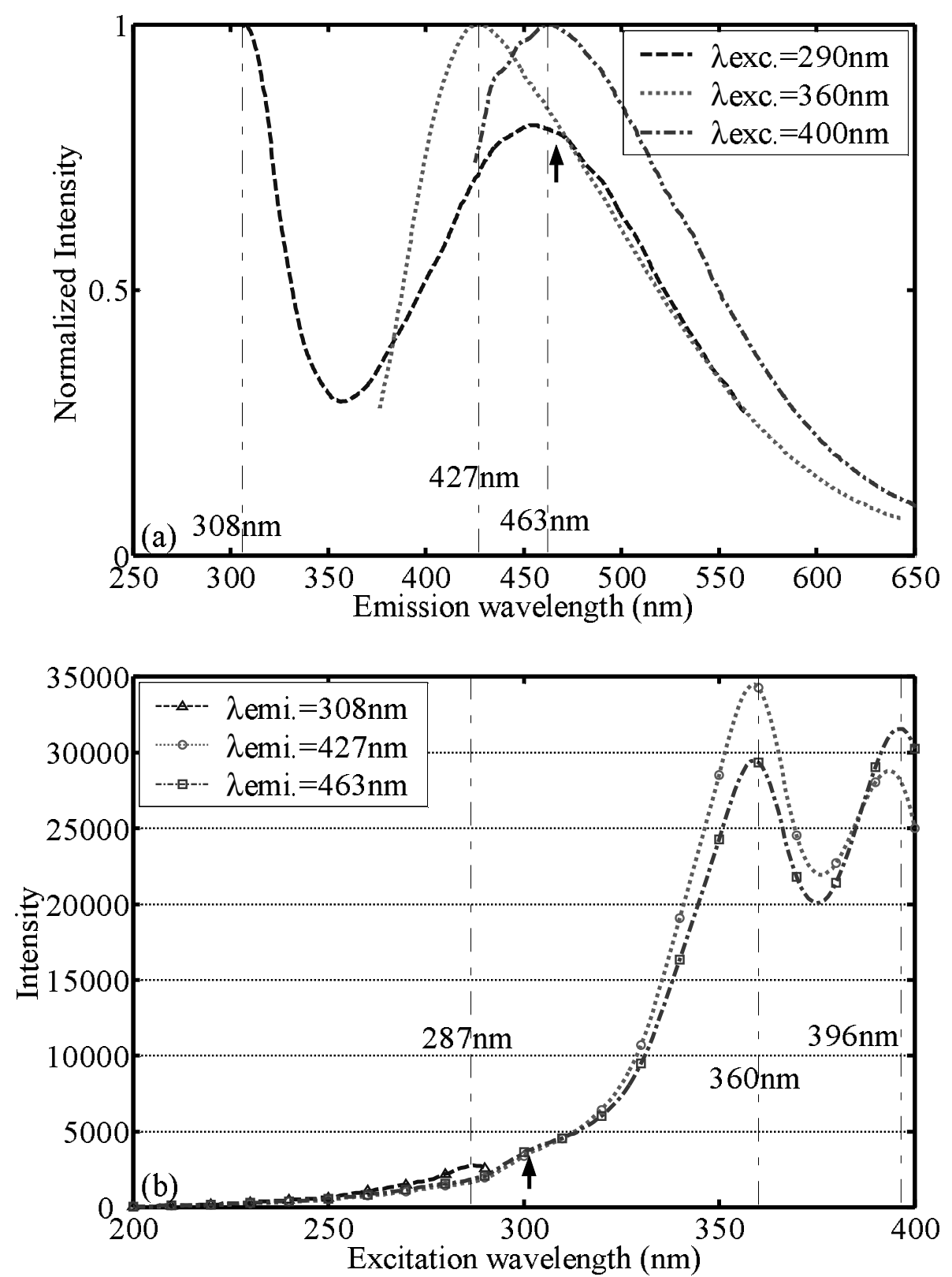

Figure 5: Low temperature photoluminescence $\left(-130^{\circ} \mathrm{C}\right)$ spectra of the ex-situ cured resin (a): Emission spectra; (b): Excitation spectra. 


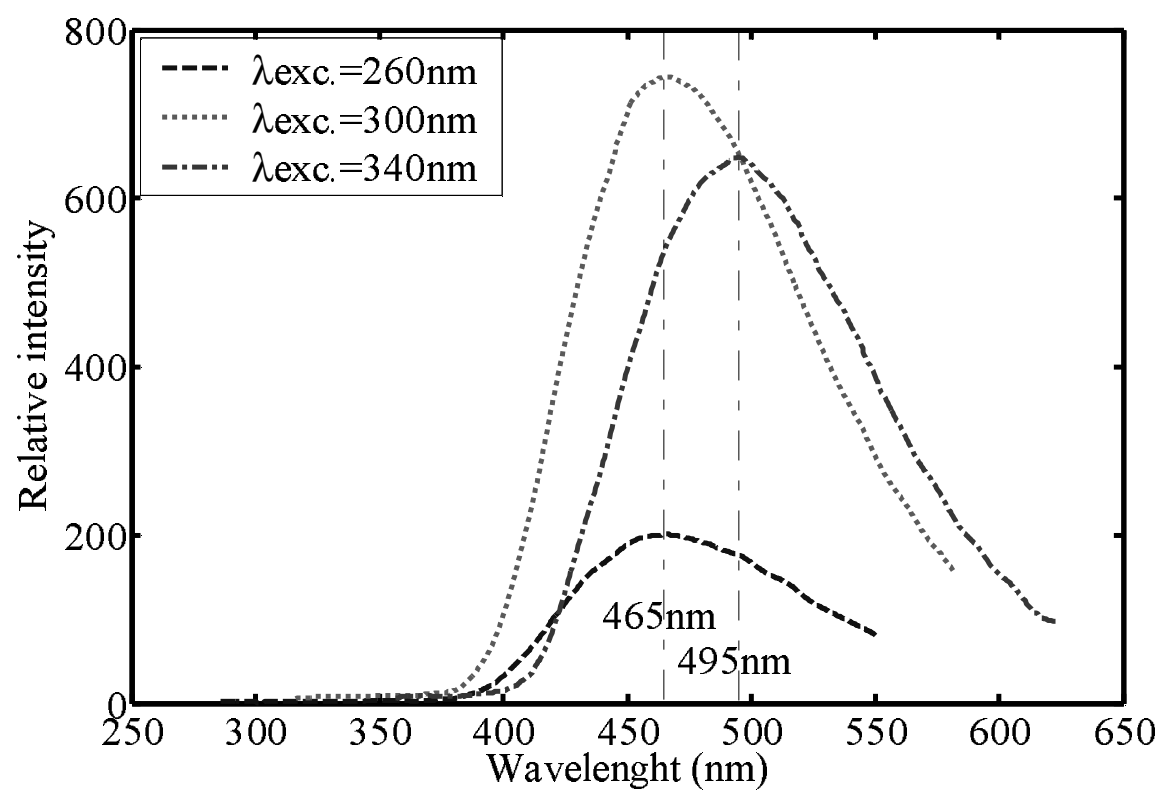

Figure 6: Low temperature phosphorescence spectra of ex-situ cured resin. 


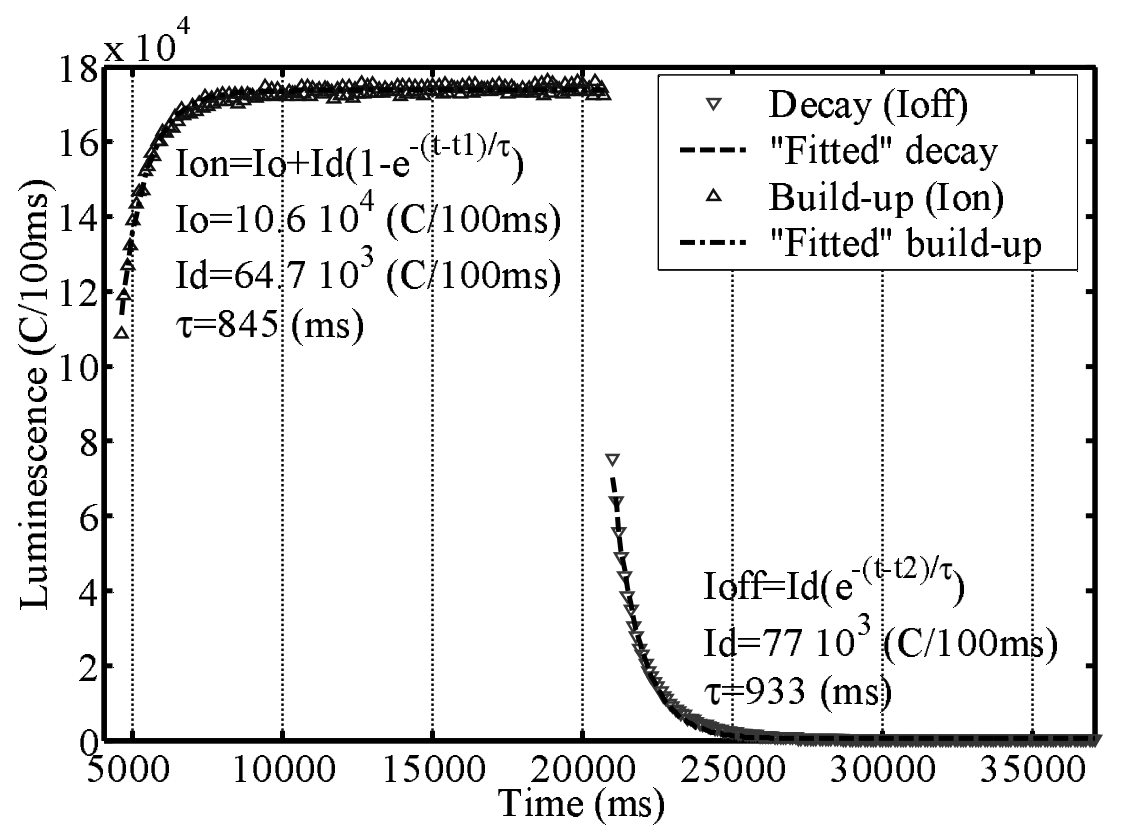

Figure 7: Luminescence transients on ex-situ cured resin after excitation at 260nm and after switching off the excitation $\left(\mathrm{T}=-130^{\circ} \mathrm{C}\right)$. The photomultiplier dwelling time was $100 \mathrm{~ms}$. Fitting parameters to Eq. 1 are given in inset. 

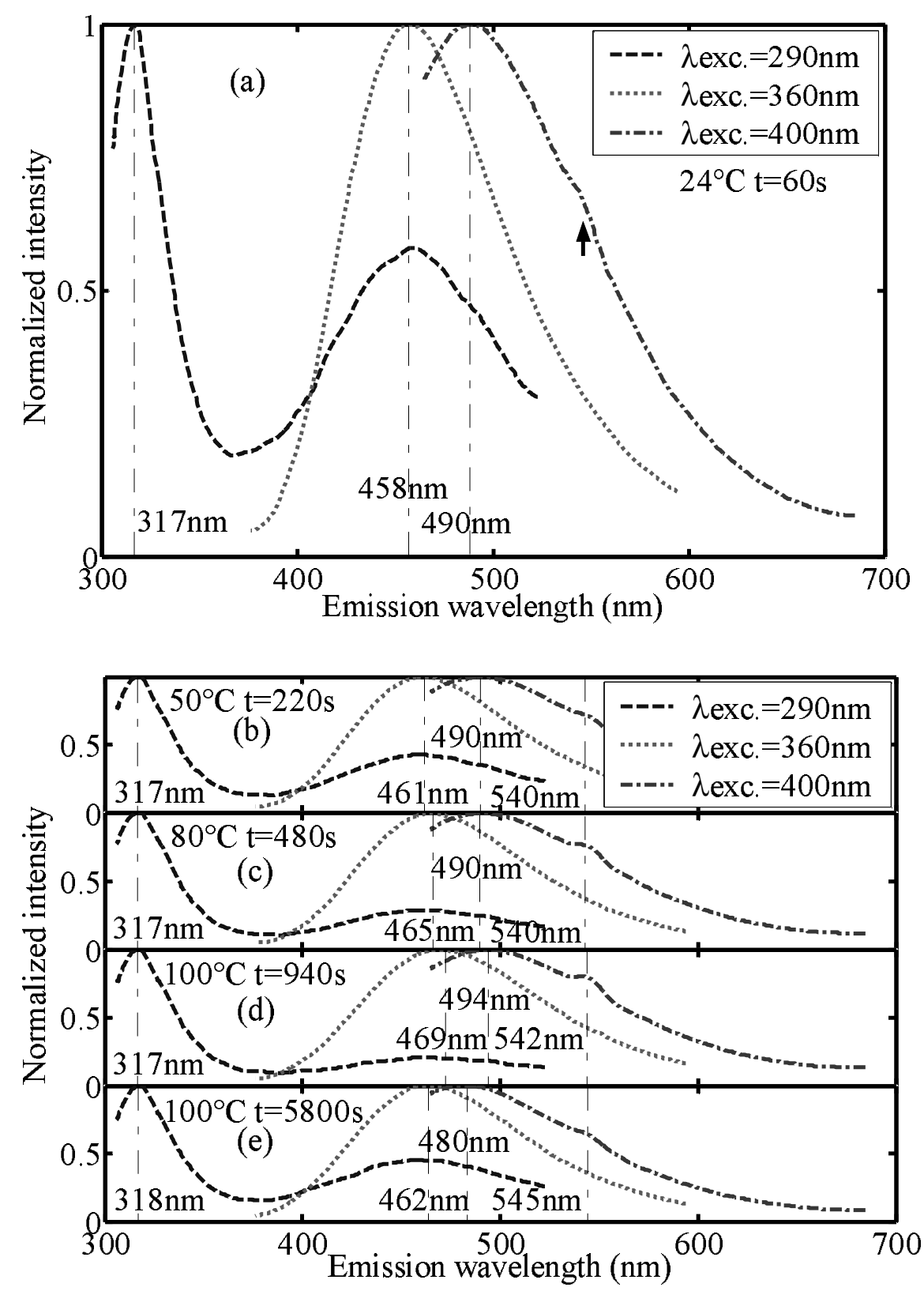

Figure 8: Normalized PL spectra recorded during in-situ curing 


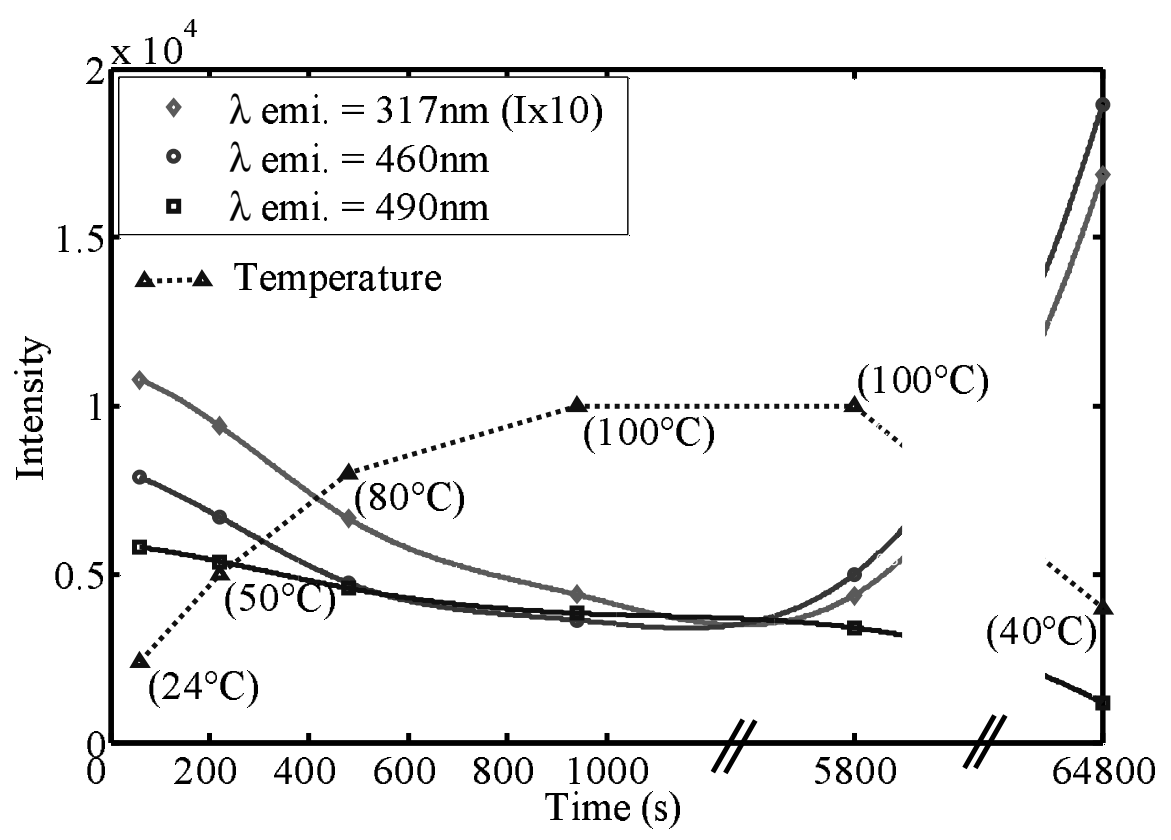

Figure 9: Emission intensities monitored at 317, 460 and 490nm for excitation respectively at 290, 360 and 400nm and corresponding temperature cycle. 


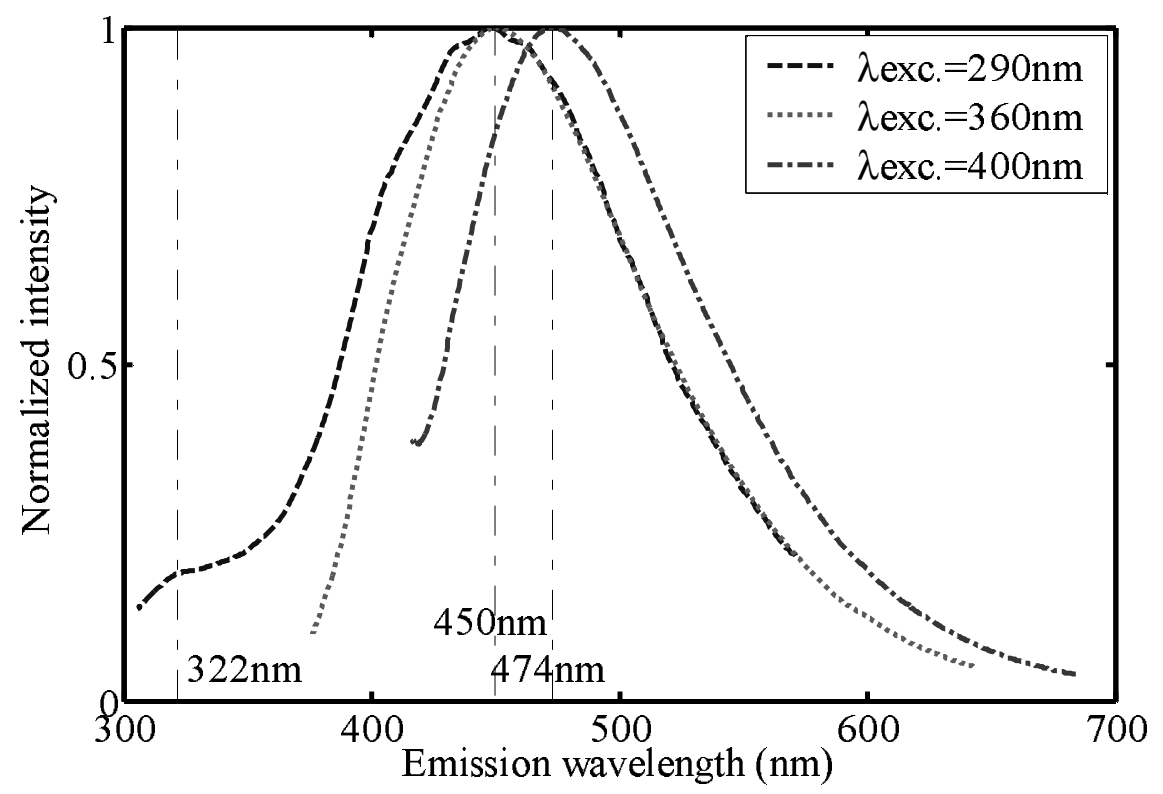

Figure 10: Normalized emission spectra of the resin cured in-situ for $18 \mathrm{~h}$ at $100^{\circ} \mathrm{C}$. 


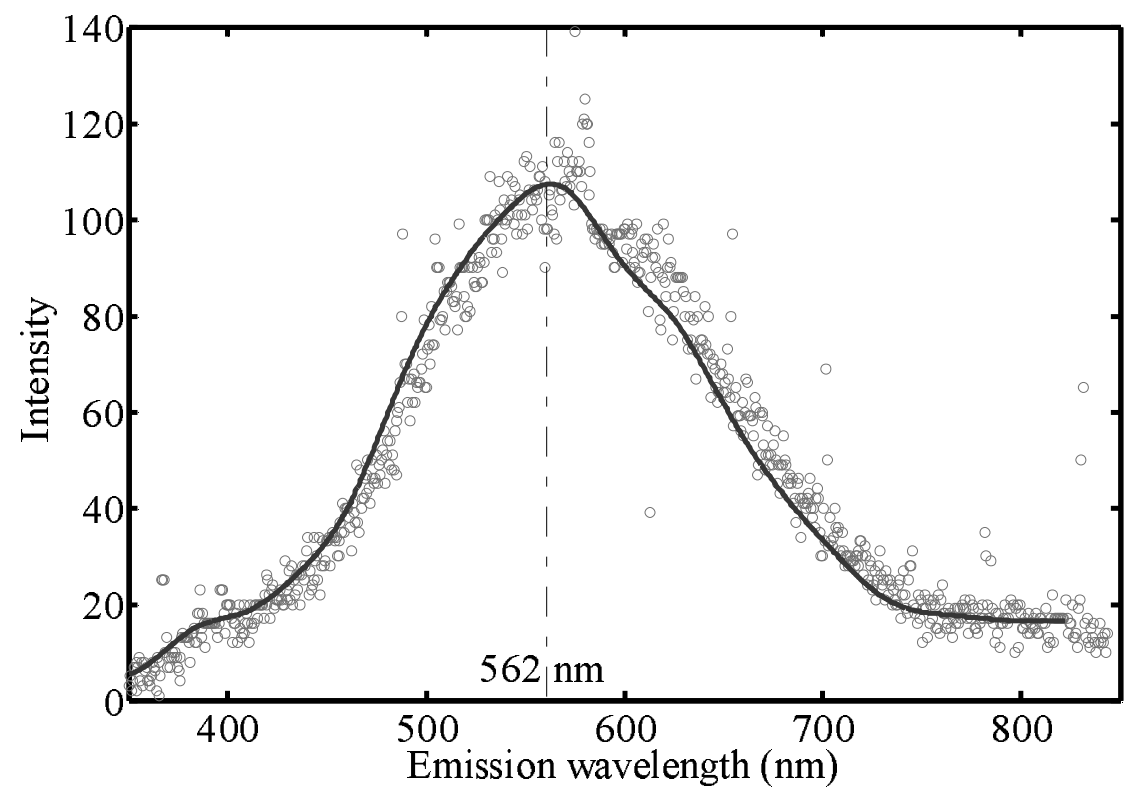

Figure 11: Spontaneous emission spectrum of the resin obtained during in-situ curing at $100^{\circ} \mathrm{C}$. The spectrum has been integrated over $5 \mathrm{~min}$ starting at the time the temperature set point was reached. The solid line is a smoothing of data. 